\title{
DISCOVERY OF X-RAY PULSATIONS FROM THE INTEGRAL SOURCE IGR J11014-6103
}

\author{
J. P. HAlPern ${ }^{1}$, J. A. Tomsick ${ }^{2}$, E. V. GotThelF ${ }^{1}$, F. CAmilo ${ }^{1}$, C.-Y. NG $^{3}$, \\ A. BodagheE ${ }^{4}$, J. RodrigueZ $^{5}$, S. ChatY $^{5,6}$, AND F. RAHOUi ${ }^{7}$ \\ ${ }^{1}$ Columbia Astrophysics Laboratory, Columbia University, 550 West 120th Street, New York, NY 10027, USA; jules@ astro.columbia.edu \\ 2 Space Sciences Laboratory, 7 Gauss Way, University of California, Berkeley, CA 94720-7450, USA \\ ${ }^{3}$ Department of Physics, The University of Hong Kong, Pokfulam Road, Hong Kong, China \\ ${ }^{4}$ Georgia College \& State University, CBX 82, Milledgeville, GA 31061, USA \\ ${ }^{5}$ Laboratoire AIM (UMR-E 9005 CEA/DSM-CNRS-Université Paris Diderot), Irfu/Service d'Astrophysique, \\ CEA-Saclay, F-91191 Gif-sur-Yvette Cedex, France \\ ${ }^{6}$ Institut Universitaire de France, 103 boulevard Saint-Michel, F-75005 Paris, France \\ ${ }^{7}$ European Southern Observatory, Karl Schwarzschild-Strasse 2, D-85748 Garching bei München, Germany \\ Received 2014 August 24; accepted 2014 October 7; published 2014 October 21
}

\begin{abstract}
We report the discovery of PSR J1101-6101, a 62.8 ms pulsar in IGR J11014-6103, a hard X-ray source with a jet and a cometary tail that strongly suggests it is moving away from the center of the supernova remnant (SNR) MSH 11-61A at $v>1000 \mathrm{~km} \mathrm{~s}^{-1}$. Two XMM-Newton observations were obtained with the EPIC pn in small window mode, resulting in the measurement of its spin-down luminosity $\dot{E}=1.36 \times 10^{36} \mathrm{erg} \mathrm{s}^{-1}$, characteristic age $\tau_{c}=116 \mathrm{kyr}$, and surface magnetic field strength $B_{s}=7.4 \times 10^{11} \mathrm{G}$. In comparison to $\tau_{c}$, the 10-30 kyr age estimated for MSH 11-61A suggests that the pulsar was born in the SNR with initial period in the range $54 \leqslant P_{0} \leqslant 60 \mathrm{~ms}$. PSR J1101-6101 is the least energetic of the 15 rotation-powered pulsars detected by INTEGRAL, and has a high efficiency of hard X-ray radiation and jet power. We examine the shape of the cometary nebula in a Chandra image, which is roughly consistent with a bow shock at the velocity inferred from the SNR age and the pulsar's $\dot{E}$. However, its structure differs in detail from the classic bow shock, and we explore possible reasons for this.
\end{abstract}

Key words: ISM: individual objects (MSH 11-61A, G290.1-0.8) - pulsars: individual (PSR J1101-6101, PSR J1105-6107) - stars: neutron - X-rays: individual (IGR J11014-6103)

\section{INTRODUCTION}

IGR J11014-6103 was discovered as a hard X-ray $(20-100 \mathrm{keV})$ source in INTEGRAL observations of the Galactic plane (Bird et al. 2010). Chandra and XMM-Newton images show that it has a complex X-ray morphology (Pavan et al. 2011; Tomsick et al. 2012; Pavan et al. 2014), consisting of a point source, a cometary pulsar wind nebula (PWN) extending 1.2 northeast of the point source, an apparent $\approx 5$.5 long jet that is oriented perpendicular to the PWN, and a faint counterjet. The PWN points back to the center of the supernova remnant (SNR) MSH 11-61A (=G290.1-0.8), and its shape and distance from the SNR suggest that the pulsar was born there and kicked with high velocity (Tomsick et al. 2012). The X-ray PWN is associated with the radio source MGPS-2 J110149-610104 detected at $843 \mathrm{MHz}$ (Pavan et al. 2011). Further mapping with the Australia Telescope Compact Array at $2 \mathrm{GHz}$ revealed that the radio source has a bow-shock morphology similar to that of the X-rays (Pavan et al. 2014).

The highly collimated, $\approx 5.5$ long $\mathrm{X}$-ray jet most likely parallels the rotation axis of the pulsar, which is therefore oriented nearly perpendicular to the velocity vector. With these properties, IGR J11014-6103 resembles the Guitar Nebula associated with the high velocity pulsar PSR B2224+65 (Hui \& Becker 2007; Hui et al. 2012; Johnson \& Wang 2010). The twisted jet (and faint counterjet) of IGR J11014-6103 were modeled by Pavan et al. (2014) as a precessing, ballistic outflow emitting synchrotron radiation.

MSH $11-61 \mathrm{~A}$ is a mixed-morphology SNR whose centrally bright, thermal X-ray emission observed by the Advanced Satellite for Cosmology and Astrophysics (ASCA) was analyzed by Slane et al. (2002) using two evolutionary models: thermal conduction and cloudy ISM. The results are that the SNR is 10-20 kyr old and is at a distance of 8-11 kpc. Since PSR $\mathrm{J} 1101-6101$ is $11^{\prime} .9$ from the center of MSH 11-61A, the evolutionary model constraints imply a tangential velocity of $v_{\perp}=2400 \mathrm{~km} \mathrm{~s}^{-1}$ and $2900 \mathrm{~km} \mathrm{~s}^{-1}$ for the thermal conduction and cloudy ISM models, respectively. (Age and distance are correlated in these models.) If this scenario is correct, PSR J1101-6101 would be the highest velocity pulsar known. However, Reynoso et al. (2006) measured a smaller distance of $7 \pm 1 \mathrm{kpc}$ to MSH 11-61 A from H $21 \mathrm{~cm}$ absorption. Using XMM-Newton and Chandra data, Garcia et al. (2012) derived an age range for MSH 11-61 A of 10-30 kyr. With these revisions, the pulsar's kick velocity is still $>800 \mathrm{~km} \mathrm{~s}^{-1}$, an exceptional value compared, e.g., to the mean two-dimensional velocity of $307 \pm 47 \mathrm{~km} \mathrm{~s}^{-1}$ for young pulsars (Hobbs et al. 2005).

\section{OBSERVATIONS AND RESULTS}

\subsection{Pulsar Discovery and Timing}

We made two XMM-Newton timing observations of IGR J11014-6103 separated by 322 days. The EPIC pn CCD was operated in small window mode, which has a $5.7 \mathrm{~ms} \mathrm{sam}$ pling time. The two EPIC MOS detectors were used in full frame mode to image the entire PWN and jet. This Letter reports only the timing results from the pn CCD. Table 1 is a log of the pn observations, indicating the net useable exposure time (elapsed, i.e., not reduced for dead-time), and the measured signal. The first observation was $44 \mathrm{ks}$ long, but its final $6 \mathrm{ks}$ were contaminated by high radiation background near perigee; thus, we use only the first $38 \mathrm{ks}$. The second observation was a clean $36.5 \mathrm{ks}$, and required no filtering. 
Table 1

XMM-Newton Timing Observations of PSR J1101-6101

\begin{tabular}{lccccccr}
\hline \hline Instr/Mode & ObsID & $\begin{array}{c}\text { Date } \\
(\mathrm{UT})\end{array}$ & $\begin{array}{c}\text { Date } \\
(\mathrm{MJD})\end{array}$ & $\begin{array}{c}\text { Exp } \\
(\mathrm{s})\end{array}$ & Counts $^{\mathrm{a}}$ & $\begin{array}{c}\text { Frequency }^{\mathrm{b}} \\
(\mathrm{Hz})\end{array}$ & $Z_{1}^{2}$ \\
\hline EPIC-pn/SW & 0722600101 & 2013 Jul 21 & 56494.033 & 38000 & 2110 & $15.9235473(14)$ & 123.5 \\
EPIC-pn/SW & 0740880201 & 2014 Jun 8 & 56816.645 & 36476 & 1997 & $15.9234868(19)$ & 68.5 \\
\hline
\end{tabular}

Notes.

a Background subtracted source counts in the $0.5-10 \mathrm{keV}$ band from a $15^{\prime \prime}$ radius aperture.

${ }^{\mathrm{b}} 1 \sigma$ uncertainty in parentheses.

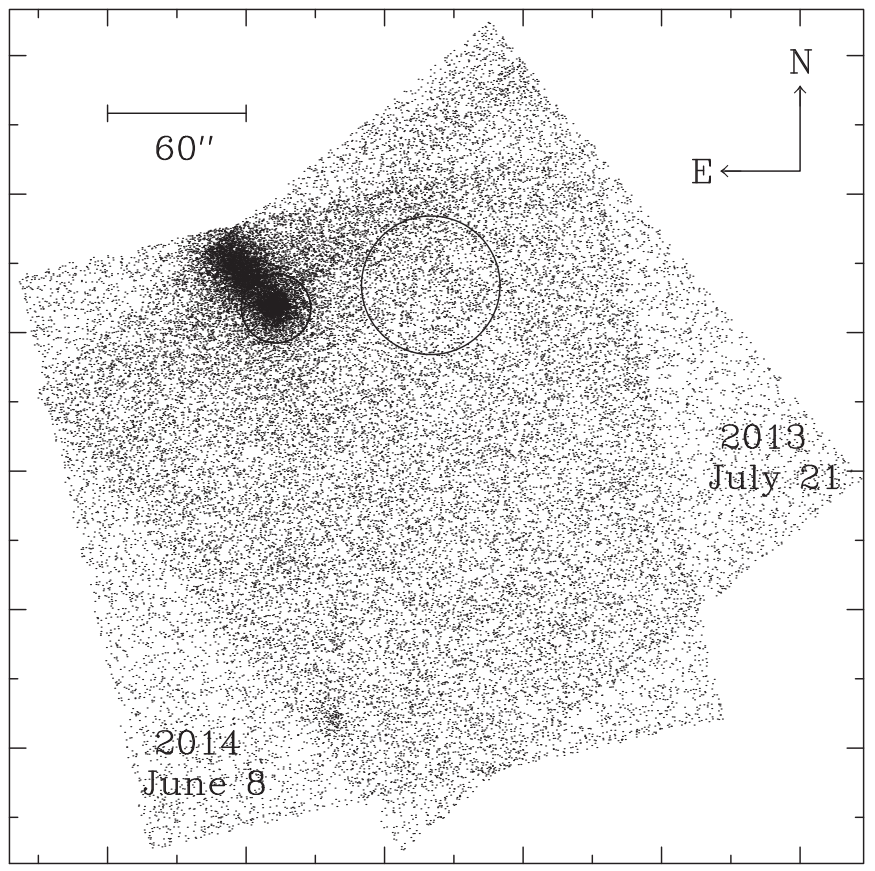

Figure 1. Two EPIC pn small window $(4 ! 3 \times 4 ! 3)$ images of IGR J11014-6103 listed in Table 1. Extraction regions are the small circle $\left(15^{\prime \prime}\right.$ radius) for PSR J1101-6101 and the large circle (30" radius) for background.

Events in the $0.5-10 \mathrm{keV}$ band were selected from a circle of radius $15^{\prime \prime}$ around the point source. This choice was a compromise between maximizing the counts extracted from the pulsar and minimizing contamination from the adjacent bowshock nebula and jet. Figure 1 shows the two images superposed, with the extraction circle for the pulsar and another circle used for background estimation. The photon arrival times were transformed to barycentric dynamical time using the Chandra measured position of the point source (Tomsick et al. 2012). The $Z_{1}^{2}$ test (Rayleigh test; Strutt 1880; Buccheri et al. 1983) was used to search for pulsations, and a single, highly significant peak was found in each observation at a period of $62.8 \mathrm{~ms}$. The $Z_{1}^{2}$ periodograms are shown in Figure 2, where the peak values are 123.5 and 68.5. Noise power $S$ is distributed as $0.5 e^{-S / 2}$, and the number of independent trials in a search to the Nyquist frequency is $\approx 3 \times 10^{6}$. This leads to negligible probabilities of $5 \times 10^{-21}$ and $4 \times 10^{-9}$, respectively, that the two detections are false. One-sigma uncertainties in the peak frequencies were estimated from the range corresponding to $Z_{1}^{2}(\max )-1$ around the peak. The significant change in frequency results in a measurement of its derivative with $6 \%$ precision. Further examination of the radio timing data reported by Tomsick et al. (2012) does not reveal a signal; thus, PSR J1101-6101 remains radio quiet to the same limit derived in Tomsick et al. (2012).

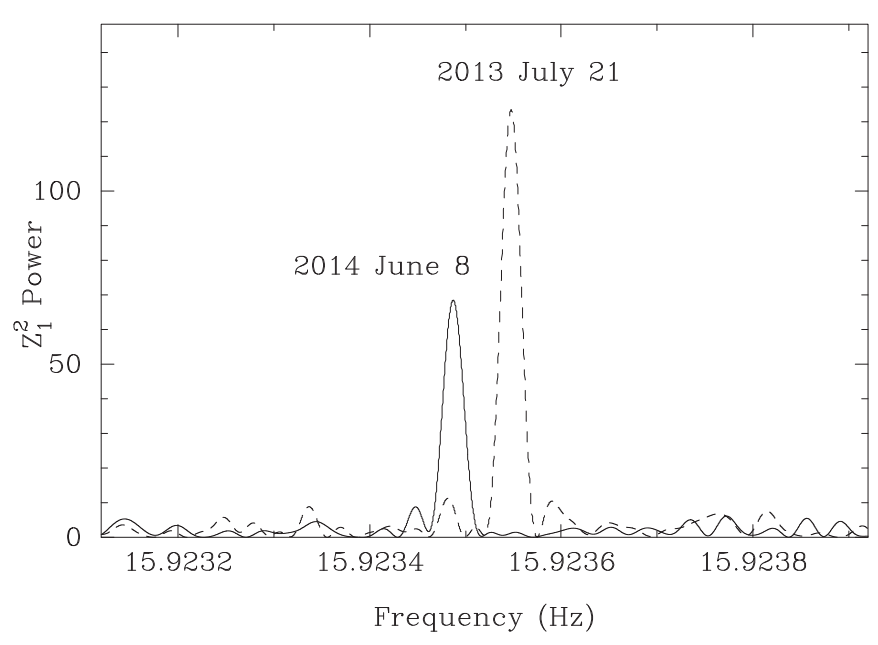

Figure 2. $Z_{1}^{2}$ periodograms from the two $X M M$-Newton timing observations listed in Table 1 . The change in frequency corresponds to $\dot{f}=(-2.17 \pm 0.13) \times$ $10^{-12} \mathrm{~s}^{-2}$.

Table 2

Timing Parameters for PSR J1101-6101

\begin{tabular}{|c|c|}
\hline Parameter & Value \\
\hline R.A. $(\mathrm{J} 2000.0)^{\mathrm{a}}$ & $11^{\mathrm{h}} 01^{\mathrm{m}} 44.96$ \\
\hline Decl. $(\mathrm{J} 2000.0)^{\mathrm{a}}$ & $-61^{\circ} 01^{\prime} 39^{\prime \prime} 6$ \\
\hline Epoch $(\text { MJD TDB })^{\mathrm{b}}$ & 56494.00000012 \\
\hline Frequency ${ }^{\mathrm{c}}, f$ & $15.9235474(14) \mathrm{s}^{-1}$ \\
\hline Frequency derivative $^{\mathrm{c}}, \dot{f}$ & $(-2.17 \pm 0.13) \times 10^{-12} \mathrm{~s}^{-2}$ \\
\hline Period $^{\mathrm{c}}, P$ & $0.062800077(6) \mathrm{s}$ \\
\hline Period derivative $^{\mathrm{c}}, \dot{P}$ & $(8.56 \pm 0.51) \times 10^{-15}$ \\
\hline Range of dates (MJD) & $56494-56817$ \\
\hline Spin-down luminosity, $\dot{E}$ & $1.36 \times 10^{36} \mathrm{erg} \mathrm{s}^{-1}$ \\
\hline Characteristic age, $\tau_{c}$ & $116 \mathrm{kyr}$ \\
\hline Surface dipole magnetic field, $B_{S}$ & $7.4 \times 10^{11} \mathrm{G}$ \\
\hline
\end{tabular}

Notes.

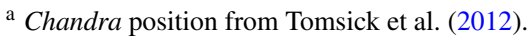

b Epoch of phase zero in Figure 3.

c $1 \sigma$ uncertainty in parentheses.

Table 2 lists the derived dipole spin-down parameters of PSR J1101-6101, including the spin-down luminosity $\dot{E}=$ $-4 \pi^{2}$ If $\dot{f}=1.36 \times 10^{36}$ erg $\mathrm{s}^{-1}$, the characteristic age $\tau_{c} \equiv|f / 2 \dot{f}|=116 \mathrm{kyr}$, and the surface dipole magnetic field strength $B_{s}=3.2 \times 10^{19}(P \dot{P})^{1 / 2} \mathrm{G}=7.4 \times 10^{11} \mathrm{G}$. An important caveat is the possibility that an intervening glitch may have biassed the measurement of $\dot{f}$. The fractional change in frequency over 322 days is $\Delta f / f=-3.8 \times 10^{-6}$. This can be compared to the largest glitches in the Vela pulsar, which have $\Delta f / f \sim 2 \times 10^{-6}$ and a mean recurrence time of $\approx 3 \mathrm{yr}$ (Espinoza et al. 2011). If PSR J1101-6101 glitched between the epochs of our observations, it is possible that its spin-down 


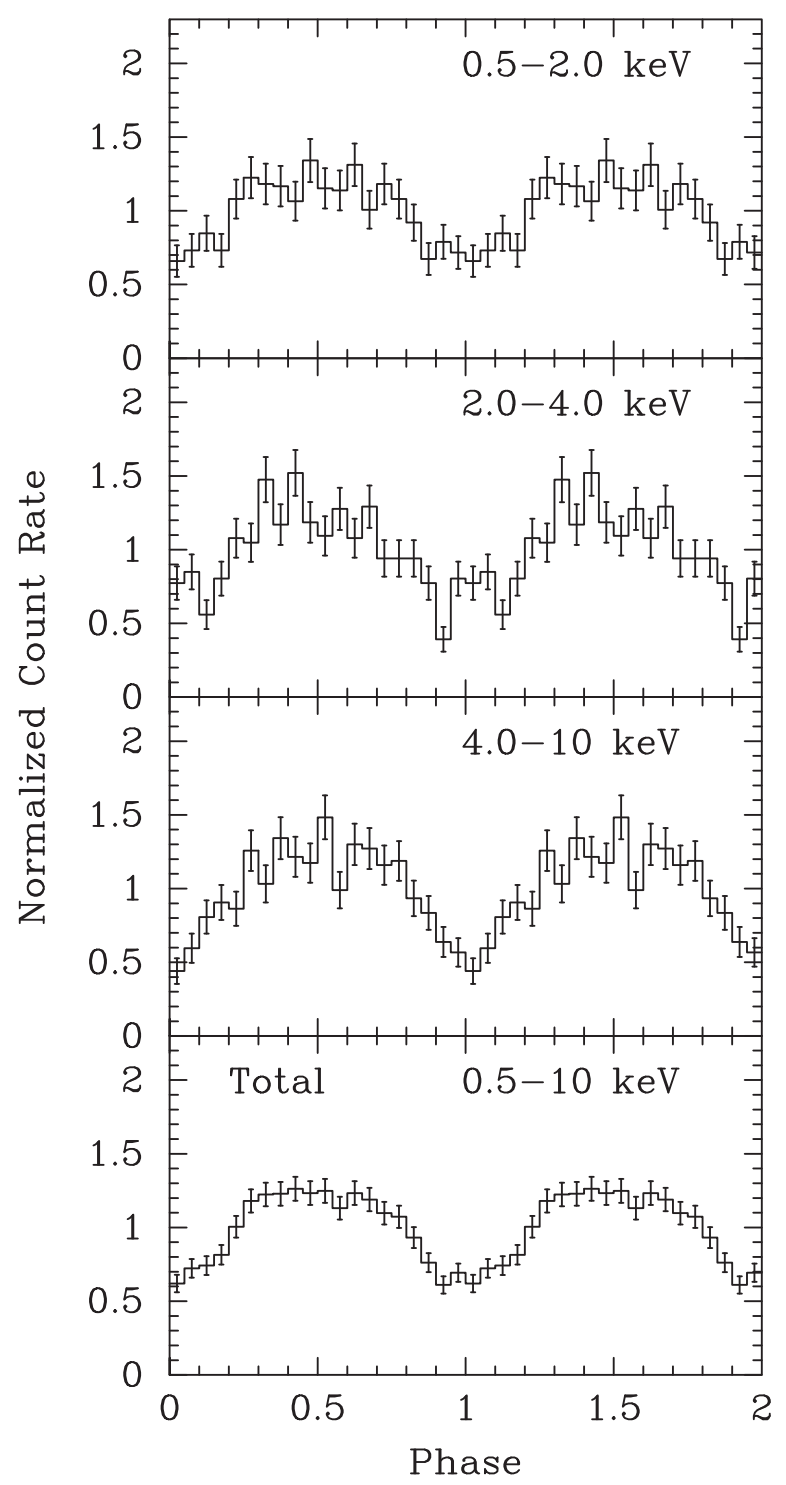

Figure 3. Energy-dependent pulse profiles of PSR J1101-6101 from the two $X M M$-Newton timing observations combined. They are background subtracted and normalized to 1 . The phase between the two observations was adjusted to align them.

rate has been underestimated by as much as $\sim 50 \%$. However, PSR J1101-6101 is not likely to be as active as the Vela pulsar, which is a uniquely strong and frequent glitcher. In any case, our main conclusions would not be changed by a $\sim 50 \%$ revision in age or spin-down power.

The difference in peak $Z_{1}^{2}$ values of the two observations, after scaling for exposure time, is not great enough to claim that the pulsed fraction has changed. The variance in measured power as a function of intrinsic power was treated by Groth (1975), and is summarized in Figure 1 of that paper (with the difference that Groth's power is actually our $Z_{1}^{2} / 2$ ). The figure shows, for example, that if the true power is $Z_{1}^{2}=100$, then there is a $16 \%$ chance that the measured power will be $>120$, and a $5 \%$ chance that it will be $<68$.

We used the timing parameters to combine the pulse profiles of the two observations, adjusting their relative phase to maximize $Z_{1}^{2}$ in the combined data. The folded light curves in a range of energies between 0.5 and $10 \mathrm{keV}$ are shown in Figure 3, where they are background-subtracted and normalized to 1 in each energy band. The single-peaked pulse shape

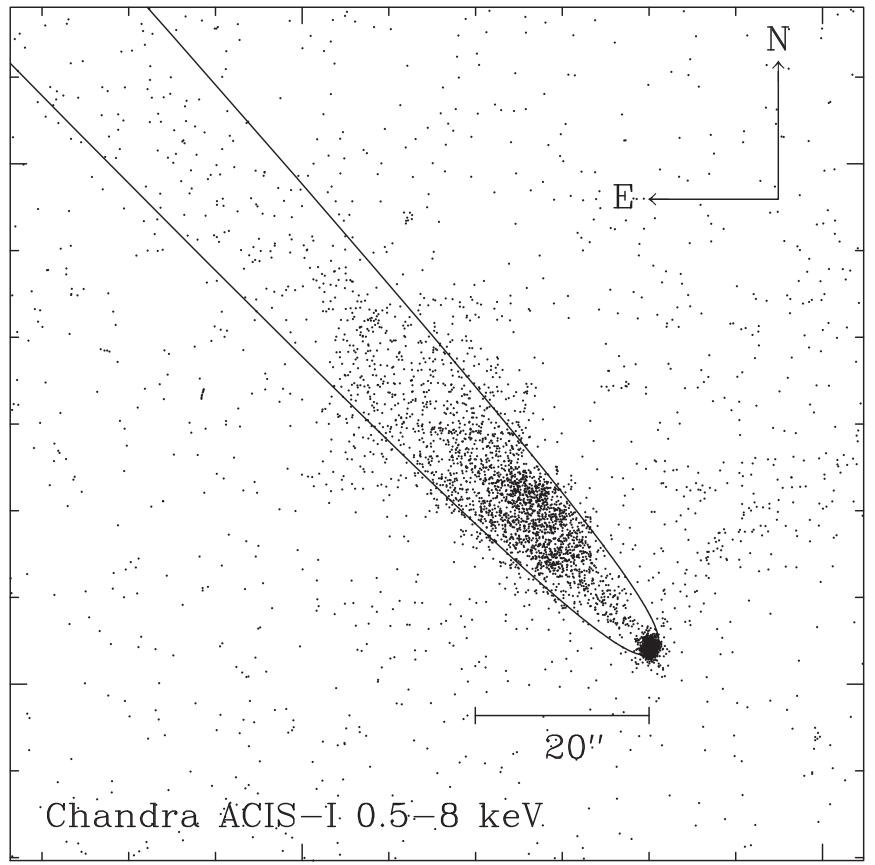

Figure 4. Chandra image of PSR J1101-6101 and its PWN from the $49.4 \mathrm{ks}$ observation of Pavan et al. (2014). Superposed is the Wilkin (1996) equation of the contact discontinuity between the shocked pulsar wind and the shocked ISM, fitted by eye. The parameters are the position angle of motion, $223^{\circ}$, and the radius of the apex, or stagnation point, which is $r_{0}=7.9 \times 10^{16} d_{7} \mathrm{~cm}\left(0 .{ }^{\prime \prime} 75\right)$ from the pulsar. The inclination angle $i$ of the velocity vector with respect to the plane of the sky is assumed to be $0^{\circ}$.

and its phasing appears to be independent of energy, while the pulsed fraction increases from $\approx 35 \%$ at the lowest energy to $\approx 50 \%$ at the highest. However, the pulsed fractions shown in Figure 3 are definitely lower limits, as the source extraction circle includes an unknown number of counts from the PWN to the northeast and, to a lesser extent, from the jet, while the circle used for background subtraction (Figure 1) does not correct for this contamination. Although the effect is difficult to quantify, it probably accounts for the apparent increase in pulsed fraction with energy in Figure 3, as the PWN has a softer spectrum than the pulsar $\left(\Gamma_{\mathrm{PWN}}=1.9 \pm 0.1, \Gamma_{\mathrm{PSR}}=1.1 \pm 0.2\right.$; Pavan et al. 2014). The intrinsic pulsed fraction is therefore likely to be $\geqslant 50 \%$ at all energies. In support of this interpretation, we find that when we decrease the radius of the extraction aperture from $15^{\prime \prime}$ to $10^{\prime \prime}$, the pulsed fraction becomes $\approx 50 \%$ at all energies.

\subsection{Bow-Shock Fitting}

Knowing the spin-down power of PSR J1101-6101, we reexamine the structure of its apparent bow-shock nebula to obtain an independent estimate of the space velocity of the pulsar. For this purpose, the higher resolution of Chandra is more useful than XMM-Newton. Our analysis here follows and extends that of Tomsick et al. (2012), who used a 5 ks Chandra observation (ObsID 12420) with the Advanced CCD Imaging Spectrometer. Here, we revisit the $49.4 \mathrm{ks}$ Chandra ACIS-I observation (ObsID 13787) that was presented by Pavan et al. (2014). Figure 4 shows the region of this image containing the pulsar and PWN, with each photon in the $0.5-8 \mathrm{keV}$ band indicated by a dot. Because the pulsar was located only 0.8 from the optical axis, the spatial resolution for the pulsar and its immediate surroundings is nearly optimal.

For the case of an isotropic wind from a star moving supersonically through a uniform ISM, Wilkin (1996) derived 
an analytic expression for the surface of contact discontinuity between the shocked pulsar wind and the shocked ISM using momentum conservation, $r(\theta)=r_{0} \csc \theta[3(1-\theta \cot \theta)]^{1 / 2}$, where $\theta$ is the polar angle with respect to direction of motion, $r(\theta)$ is the distance of the surface from the star, and $r_{0}$ is the stagnation radius, the distance of the apex of the surface from the pulsar. The shape of the contact discontinuity is thus parameterized entirely in terms of $r_{0}$, which in turn is determined in this case by the pulsar wind power, assumed to be $\approx \dot{E}$, the velocity $v$ of the pulsar, and the ambient density $\rho$ of the ISM, using momentum balance:

$$
r_{0}=\left(\frac{\dot{E}}{4 \pi \rho v^{2} c}\right)^{1 / 2} .
$$

The assumption we make in graphing the model curve in Figure 4 is that the X-ray emission comes from the shocked pulsar wind, which is bounded by the contact discontinuity and the termination shock interior to it. Therefore, $r_{0}$ was chosen by eye so that the curve surrounds the bulk of the emission trailing the pulsar. It was assumed that the pulsar is moving nearly in the plane of the sky $\left(i=0^{\circ}\right)$, both because of the narrow opening angle of the nebula, and the evidently large tangential velocity. The parameters of the curve are $r_{0}=7.9 \times 10^{16} d_{7} \mathrm{~cm}\left(0 .{ }^{\prime \prime} 75\right)$, where $d_{7}$ is the distance in units of $7 \mathrm{kpc}$, and the position angle of the motion, $223^{\circ}$. Similar results were obtained by Tomsick et al. (2012). Now substituting the values of $r_{0}$ and $\dot{E}$, Equation (1) is reduced to $v_{\perp}=500 d_{7}^{-1} n_{0.1}^{-1 / 2} \mathrm{~km} \mathrm{~s}^{-1}$, where $n_{0.1}$ is the ISM hydrogen density in units of $0.1 \mathrm{~cm}^{-3}$. This velocity is $\sim 2-4$ times less than the estimates from the SNR age, but they can be reconciled if the local density is $\sim 0.01 \mathrm{~cm}^{-3}$. The result is largely unaffected by the unknown angle $i$, because any inclination of the model would broaden the apparent opening angle of the bow shock, which would then have to be reduced by decreasing $r_{0}$, thus increasing $v$.

However, before giving this analysis too much credence, note that there are at least two discrepancies between the simple model and the detailed properties of the data. First, there is no evidence of emission from the apex of the shocked wind, which theoretically should be the brightest part of the nebula. In fact, there is no excess emission within $\approx 3^{\prime \prime}$ of the pulsar, a zone that is entirely consistent with a single point source (Tomsick et al. 2012; Pavan et al. 2014). Second, the faint X-ray emission just behind the pulsar does not in fact follow the model curve, but is confined to a narrower cone, while the brightest regions filling the curve are $13^{\prime \prime}-26^{\prime \prime}$ behind the pulsar. The appearance is of a diverging flow getting brighter with distance from the pulsar, rather than a collimated one that is fading. Similar phenomena have been noted in the X-ray images of other pulsar tails; the possible implications will be discussed in Section 3.3.

\section{DISCUSSION}

\subsection{Distance and Associations}

Various estimates of the distance to MSH 11-61A were reviewed by Filipović et al. (2005), who concluded from their own CO maps that $d=7-8 \mathrm{kpc}$, in agreement with $6.9 \mathrm{kpc}$ from optical emission-line velocities (Rosado et al. 1996), and $7 \pm 1 \mathrm{kpc}$ from Hi $21 \mathrm{~cm}$ absorption (Reynoso et al. 2006). These are all kinematic distances, unlike the X-ray modelling of Slane et al. (2002), who derived $d=8-11 \mathrm{kpc}$. We have adopted $7 \mathrm{kpc}$ as the most likely distance.
Since the characteristic age of PSR J1101-6101 is greater than all estimates of the age of MSH 11-61A, we can assume that it was born in the SNR and estimate its birth period $P_{0}$ from the relation

$$
T=\frac{P}{(n-1) \dot{P}}\left[1-\left(\frac{P_{0}}{P}\right)^{n-1}\right],
$$

where $n \equiv f \ddot{f} / \dot{f}^{2}$ is the braking index. Most pulsars have $2<n<3$ (Livingstone et al. 2007). For this range, and letting $T$ be the $10-30 \mathrm{kyr}$ age of the SNR, we find $54 \leqslant P_{0} \leqslant 60 \mathrm{~ms}$.

PSR J1101-6101 should not be confused with PSR J1105-6107, a $63.2 \mathrm{~ms}$ pulsar with $\dot{E}=2.5 \times 10^{36} \mathrm{erg} \mathrm{s}^{-1}$ and $\tau_{c}=63 \mathrm{kyr}$ that is $23^{\prime}$ southeast of MSH 11-61A. Kaspi et al. (1997) considered the possibility that PSR J1105-6107 was born in MSH 11-61A, although PSR J1101-6101 is now a more compelling association. The dispersion measure of $271 \mathrm{pc} \mathrm{cm}^{-3}$ to PSR J1105-6107 converts to a distance of $5.0 \mathrm{kpc}$ according to the Galactic electron density model of Cordes \& Lazio (2002). The corresponding free-electron column of $N_{\mathrm{e}}=8.4 \times 10^{20} \mathrm{~cm}^{-2}$, assuming a typical ionized fraction of 0.1 (He et al. 2013), is accompanied by a neutral column of $N_{\mathrm{H}} \approx 8.4 \times 10^{21} \mathrm{~cm}^{-2}$, which is consistent with the X-ray measured $N_{\mathrm{H}}=8 \times 10^{21} \mathrm{~cm}^{-2}$ to IGR J11014-6103 (Tomsick et al. 2012; Pavan et al. 2014). X-ray measurements of $N_{\mathrm{H}}$ to MSH 11-61A are somewhat contradictory, ranging from $(4.3-6.2) \times 10^{21} \mathrm{~cm}^{-2}$ (Garcia et al. 2012) to $(1.3 \pm 0.1) \times 10^{22} \mathrm{~cm}^{-2}$ (Slane et al. 2002). Allowing for this ambiguity, all three objects are probably consistent with being at the same distance.

PSR J1105-6107 is not detected in X-rays. Using archival Chandra observations totaling $23.7 \mathrm{ks}$ (ObsIDs 2780 and 4380), we set a $3 \sigma$ upper limit of $8 \times 10^{-15} \mathrm{erg} \mathrm{cm}^{-2} \mathrm{~s}^{-1}$ on its $2-10 \mathrm{keV}$ flux, corresponding to $L_{X} / \dot{E}<2 \times 10^{-5} d_{7}^{2}$. This is close to the minimum of the distribution of similarly aged pulsars (Kargaltsev \& Pavlov 2008). A previously claimed detection of this pulsar using ASCA (Gotthelf \& Kaspi 1998) may have instead detected a neighboring star that is present in the Chandra images.

\subsection{Energetics}

With $\dot{E}=1.36 \times 10^{36} \mathrm{erg} \mathrm{s}^{-1}$, PSR J1101-6101 is the least energetic of the 15 rotation-powered pulsars detected by INTEGRAL (for the full set see Mattana et al. 2009; Renaud et al. 2010; Gotthelf et al. 2011; Halpern et al. 2012). These are among the most energetic pulsars, comprising half of all those known with $\dot{E} \geqslant 3.7 \times 10^{36} \mathrm{erg} \mathrm{s}^{-1}$, the latter value belonging to PSR B1951+32, which has a characteristic age of $107 \mathrm{kyr}$ and was previously the least energetic of the INTEGRAL pulsars.

The $20-100 \mathrm{keV}$ flux of IGR J11014-6103 is $8.7 \times$ $10^{-12} \mathrm{erg} \mathrm{cm}^{-2} \mathrm{~s}^{-1}$ (Bird et al. 2010), corresponding to a luminosity of $5.1 \times 10^{34} d_{7}^{2} \mathrm{erg} \mathrm{s}^{-1}$ that is $4 \%$ of the spin-down luminosity of PSR J1101-6101. This exceeds the combined $2-10 \mathrm{keV}$ flux of the pulsar, the PWN, and the jet as measured by Chandra, which total $1.8 \times 10^{-12} \mathrm{erg} \mathrm{cm}^{-2} \mathrm{~s}^{-1}$ (Pavan et al. 2014 ), or $0.8 \%$ of the spin-down flux. The flat spectrum of the pulsar must extend into the hard X-rays, where it is responsible for most of the 20-100 keV flux. Using equipartition arguments, Pavan et al. (2014) estimated that a minimum power of $2 \times 10^{35}$ erg s $\mathrm{s}^{-1}$ is needed for the jet, which is $14 \%$ of the spin-down luminosity. This is important evidence that a large part of a pulsar's spin-down power can be focused into a narrow polar jet, a fraction therefore not available to power a bow shock. 


\subsection{Structure of the PWN}

The absence of X-rays from the head of the putative bow shock is the principal challenge to the model in which the termination shock of the pulsar is the cause of the PWN emission. A dark region between the termination shock and the contact discontinuity is difficult to understand in the context of shock acceleration. For reasonable values of the magnetic field strength $\left(B \sim 10^{-4} \mathrm{G}\right)$ and $\mathrm{X}$-ray emitting electron energy ( $E \sim 10^{13} \mathrm{eV}$ ), the gyroradius, $r_{g}=3 \times 10^{14} E_{13} B_{-4}^{-1} \mathrm{~cm}$, is much smaller than the stagnation radius, $r_{0}=7.9 \times 10^{16} d_{7} \mathrm{~cm}$. So the particles should be easily confined and accelerated.

The cavity interior to the termination shock should be dark, so that any emission from around it should be limb brightened. The termination shock will close behind the pulsar at a distance $r_{1}$, which is larger than its forward radius, $\approx r_{0}$. In analytic and numerical models at low Mach number $\mathcal{M}$, the relation between these radii is $r_{1} / r_{0} \approx \gamma^{1 / 2} \mathcal{M}$, where $\gamma$ is the adiabatic index of the ambient medium, is usually $5 / 3$ (Bucciantini 2002). However, numerical models at high $\mathcal{M}$ show that this ratio saturates at about 5 (Gaensler et al. 2004). While an area $\approx 12^{\prime \prime}$ long behind PSR J1101-6101 is relatively dim in Xrays, the emission there looks like a narrow cone rather than the expected limb-brightened bow shock. Nevertheless, if we ignore this detail and assume that this is the region bounded by the termination shock, then $r_{1} / r_{0} \approx 12^{\prime \prime} / 0$."75 and $\mathcal{M} \approx 12.4$. The sound speed in the warm $(8000 \mathrm{~K})$ phase of the ISM is $\approx 13$ $\mathrm{km} \mathrm{s}^{-1}$, which then implies a pulsar velocity of only $\approx 165 \mathrm{~km}$ $\mathrm{s}^{-1}$, at odds with the other estimates. Only if the ISM is hot $\left(\sim 10^{6} \mathrm{~K}\right)$ do we get $v \approx 1900 \mathrm{~km} \mathrm{~s}^{-1}$. But this would require reducing the ambient density drastically to have a reasonable pressure, which would be inconsistent with the results from Equation (1).

Gaensler et al. (2004) suggested that the "tongue" of emission just behind PSR J1747-2958 (the Mouse) and others represents the surface of the termination shock. But these are bright regions, which contradicts the theory that there should be no emission interior to the termination shock. In the case of PSR $\mathrm{J} 1101-6101$, this region is at least underluminous, although not limb-brightened. Another system like PSR J1101-6101 in which trailing emission brightens with distance from the pulsar is PSR J0357+3205 (De Luca et al. 2011, 2013; Marelli et al. 2013). The difficulties in modeling that tail as a synchrotron emitting bow shock led the authors to propose shocked-heated bremsstrahlung emission instead. But that model requires a hot ISM phase with an extraordinarily large pressure.

In several pulsar tails, radio and X-ray brightness are anticorrelated, with the radio increasing with distance from the pulsar (Ng et al. 2010). This is the case for PSR J1101-6101 as well (Pavan et al. 2014). However, it is not clear if this phenomenon relates to why the region closest to PSR J1101-6101 is underluminous in both radio and X-ray.

Considering that the spin axis of PSR J1101-6101 may be orthogonal to its velocity vector, with a large fraction of the spindown power going into the jet, one may ask if the remaining wind is primarily polar or equatorial, and how that would affect the structure of the PWN. However, numerical models with anisotropic pulsar winds, including an equatorial one, do not significantly change the shape of the termination shock (Vigelius et al. 2007). So far, no model within the framework of ideal MHD appears to explain the features of our data and others.

An alternative model (Romanova et al. 2005) in which particles are accelerated by magnetic reconnection outside the speed-of-light cylinder results in a fast "magnetotail" behind the pulsar, which may contain a large fraction of the energy of the pulsar wind and extend to large distances. An interesting feature of this model is the flared "trumpet" shape of the magnetotail (Figure 4 of Romanova et al. 2005), which does in fact resembles the PWN of PSR J1101-6101. An approximation for the radiation length of the magnetotail is

$$
r \approx 15 P^{2}\left(\frac{B_{s}}{10^{12} \mathrm{G}}\right)^{-1}\left(\frac{n}{\mathrm{~cm}^{-3}}\right)^{-1}\left(\frac{v}{1000 \mathrm{~km} \mathrm{~s}^{-1}}\right)^{-2} \mathrm{pc} .
$$

Assuming $n=0.03 \mathrm{~cm}^{-3}$ and $v=1000 \mathrm{~km} \mathrm{~s}^{-1}$, this reduces to $r \approx 2.7 \mathrm{pc}(1.3)$, the actual length of the PWN. However, the authors only investigated the case in which the magnetic axis, the rotation axis, and the velocity are all parallel, while there is good reason to believe that the rotation axis of PSR J1101-6101 is nearly orthogonal to its velocity because of the orientation of the jet. It is not clear if a more realistic geometry would generate undesirable, nonaxisymmetric features.

\section{CONCLUSIONS}

We discovered the $62.8 \mathrm{~ms}$ pulsar PSR J1101-6101 in IGR J11014-6103. Its spin-down luminosity of $1.36 \times$ $10^{36} \mathrm{erg} \mathrm{s}^{-1}$ is the lowest among the 15 rotation-powered pulsars detected by INTEGRAL, and an order-of-magnitude less than what was anticipated from the X-ray luminosity of PSR J1101-6101 (Pavan et al. 2014). However, there is a large scatter among pulsars in efficiency of X-ray emission. Its $116 \mathrm{kyr}$ characteristic age is consistent with an origin in MSH 11-61 A for any reasonable value of the braking index or SNR age, with its birth period close to its present period.

The velocity of the pulsar inferred from fitting the shape of its cometary nebula is compatible with estimates of $800-2400 \mathrm{~km} \mathrm{~s}^{-1}$ from the SNR age and distance, if the density of the ambient ISM is $<0.1 \mathrm{~cm}^{-3}$. The density should be this low if PSR J1101-6101 is within a cavity blown by previous stellar winds or supernovae. Because the structure of the nebula differs in important details from a basic bow-shock geometry, we are not secure in making quantitative estimates of velocity and density from such a simple model. Interestingly, however, an alternative magnetotail theory would require similar velocity and density.

\section{REFERENCES}

Bird, A. J., Bazzano, A., Bassani, L., et al. 2010, ApJS, 186, 1 Bucciantini, N. 2002, A\&A, 387, 1066

Buccheri, R., Bennett, K., Bignami, G. F., et al. 1983, A\&A, 128, 245 Cordes, J. M., \& Lazio, T. J. W. 2002, arXiv:astro-ph/0207156 De Luca, A., Marelli, M., Mignani, R. P., et al. 2011, ApJ, 733, 104 De Luca, A., Mignani, R. P., Marelli, M., et al. 2013, ApJL, 765, L19 Espinoza, C. M., Lyne, A., Stappers, B. W., \& Kramer, M. 2011, MNRAS, 414, 1679

Filipović, M. D., Payne, J. L., \& Jones, P. A. 2005, SerAJ, 170, 47

Gaensler, B. M., van der Swaluw, E., Camilo, F., et al. 2004, ApJ, 616, 383

Garcia, F., Combi, J. A., Albacete-Colombo, J. F., et al. 2012, A\&A, 546, A91

Gotthelf, E. V., Halpern, J. P., Terrier, R., \& Mattana, F. 2011, ApJL, 729, L16 Gotthelf, E. V., \& Kaspi, V. M. 1998, ApJL, 497, L29

Groth, E. J. 1975, ApJS, 29, 285

Halpern, J. P., Gotthelf, E. V., \& Camilo, F. 2012, ApJL, 753, L14

He, C., Ng, C.-Y., \& Kaspi, V. M. 2013, ApJ, 768, 64

Hobbs, G., Lorimer, D. R., Lyne, A. G., \& Kramer, M. 2005, MNRAS, 360,974

Hui, C. Y., \& Becker, W. 2007, A\&A, 467, 1209

Hui, C. Y., Huang, R. H. H., Trepl, L., et al. 2012, ApJ, 747, 74

Johnson, S. P., \& Wang, Q. D. 2010, MNRAS, 408, 1216 
Kargaltsev, O., \& Pavlov, G. G. 2008, in AIP Conf. Proc. 983, 40 Years of Pulsars: Millisecond Pulsars, Magnetars and More, ed. C. Bassa, Z. Wang, A. Cumming, \& V. M. Kaspi (Melville, NY: AIP), 171

Kaspi, V., Bailes, M., Manchester, R. N., et al. 1997, ApJ, 485, 820

Livingstone, M. A., Kaspi, V. M., Gavriil, F. P., et al. 2007, Ap\&SS, 308, 317

Marelli, M., De Luca, A., Salvetti, D., et al. 2013, ApJ, 765, 36

Mattana, F., Götz, D., Terrier, R., Renaud, M., \& Falanga, M. 2009, in AIP Conf. Proc. 1126, Simbol-X: Focusing on the Hard X-ray Universe, ed. J. Rodriguez \& P. Forrando (Melville, NY: AIP), 259

Ng, C.-Y., Gaensler, B. M., Chatterjee, S., \& Johnston, S. 2010, ApJ, 712, 596

Pavan, L., Bordas, P., Pühlhofer, G., et al. 2014, A\&A, 562, A122

Pavan, L., Bozzo, E., Pühlhofer, G., et al. 2011, A\&A, 533, A74
Renaud, M., Marandon, V., Gotthelf, E. V., et al. 2010, ApJ, 716, 663

Reynoso, E. M., Johnston, S., Green, A. J., et al. 2006, MNRAS, 369, 416

Romanova, M. M., Chulsky, G. A., \& Lovelace, R. V. E. 2005, ApJ, 630, 1020

Rosado, M., Ambrocio-Cruz, P., Le Coarer, E., \& Marcelin, M. 1996, A\&A, 315,243

Slane, P., Smith, R. K., Hughes, J. P., \& Petre, R. 2002, ApJ, 564, 284

Strutt, J. W. 1880, PMag, 10, 73

Tomsick, J. A., Bodaghee, A., Rodriguez, J., et al. 2012, ApJL, 750, L39

Vigelius, M., Melatos, A., Chatterjee, S., Gaensler, B. M., \& Ghavamian, P. 2007, MNRAS, 374, 793

Wilkin, F. P. 1996, ApJL, 459, L31 